\title{
IMPACT OF ANTIDEPRESSANT DULOXETINE ON THE LINGUAL MUCOSA OF MALE ALBINO RATS (AN ULTRASTRUCTURAL, AND IMUNOHISTOCHEMICAL STUDY)
}

\author{
Rasha Mohamed Taha* and Rania Hanafi Mahmoud**
}

\begin{abstract}
Objectives: This study was designed to clarify the effect of Duloxetine on the lingual mucosa of male albino rats.

Materials and methods: Forty male albino rats divided as: 20 animals served as control. Group II: 20 animals and received duloxetine in a dose of $10 \mathrm{mg} / \mathrm{kg}$ body weight that dissolved in distilled water and administrated through the metallic curved oropharngeal tube. At the end of the experiments, which lasts for 3 months, half of the animals of different groups (group I.1 and II.1) were sacrificed. The rest of animals (group I.2 and II.2) were allowed for a recovery period of 30 days. the tongue of all groups were extracted and prepared to examined through histological examination of their lingual mucosa to detect any structural changes, transmission electron microscopic examination of their lingual mucosa to detect any ultrastructural changes and immunohistochemical localization of E-cadherin
\end{abstract}

Results: The light microscopic examination of the tongue of the rats treated with Duloxetine, anti-depressant drug, showed degenerative changes that involved the surface epithelium and lamina propria. The animals left for a recovery period showed partial improvement in their structure, these results were indicated by ultrastructural results. The immunohistochemical results of Duloxetine group showed weakly positive reactivity of E- cadherin while the recovery group showed moderately positive reactivity to E cadherin.

Conclusion: Duloxetine has a degenerative effect upon the experimental level.

KEY WORD: Duloxetine, anti-depressant, Anxiety disorders, selective serotonin reuptake inhibitors drugs, lingual mucosa, E-cadherin

* Lecturer of Oral Biology Department, Faculty of Dentistry, Suez Canal University

** Associated Professor of Oral Pathology Department, Faculty of Dentistry, Suez Canal University 


\section{INTRODUCTION}

Anxiety disorders are common in children and adolescents with reported prevalence rates between $2.4 \%$ and $29.8 \%{ }^{1}$. It has a strong association with personal distress, impaired social and occupational functions, hampered quality-of-life, and overall substantial economic $\operatorname{loss}^{2}$. Baldwin et al. ${ }^{3}$ areported substantial unmet public health, clinical, and research needs in the intervention of anxiousness disorders. In children and adolescents who might benefit from use of medications, selective serotonin reuptake inhibitors (SSRIs) are alternative drugs ${ }^{4}$. Antidepressants drugs such as duloxetine serotonin and norepinephrine reuptake inhibitors (SNRIs), is approved for the treatment of generalized anxiety disorders. Duloxetine (SNRIs) block both the serotonin and norepinephrine reuptake and weakly inhibit dopamine reuptake leading to increased serotonergic, noradrenergic, and dopaminergic neurotransmission ${ }^{5}$. Side effects of SNRIs are similar to those of selective serotonin reuptake inhibitors (SSRIs) but also includes an increase in systemic blood pressure, especially dose-dependent increase in supine systolic and diastolic blood pressure has been reported ${ }^{6}$. Hyponatremia and syndrome of inappropriate vasopressin secretion are uncommon however are reportable with SNRIs 7. Nervousness, somnolence, nausea, decreased appetite, weight loss, constipation, increased sweating, dry mouth, dizziness, troubled sleeping and sexual dysfunction are the most commonly reported side effects of duloxetine ${ }^{5}$.

Duloxetine was recommended as a first choice for the treatment of chemotherapy-induced neuropathy by the American Society of Clinical Oncol$\mathrm{ogy}^{8}$, as a best therapy for fibromyalgia treatment in the presence of mood disorders by the German Interdisciplinary Association for Pain Therapy ${ }^{9}$, as a Grade B recommendation for the treatment of diabetic neuropathy and fibromyalgia by the American Association for Neurology ${ }^{10}$ and as a level A recom- mendation in certain neuropathic states by the European Federation of Neurological Societies ${ }^{11}$.

The most commonly reported treatment -emergent adverse events among duloxetine-treated patient were nausea, dry mouth, headache and dizziness $^{12}$, long-term subject area of fibromyalgia patients receiving duloxetine, frequency and type of adverse issue was similar. Incline effects tended to be mild-to-moderate, and tended to be reduced in intensity over time ${ }^{13}$.

Duloxetine inhibits the re-uptake of 5-hydroxytryptamine and norepinephrine in the central nervous arrangement, while increases dopamine (District attorney) specifically in the prefrontal cerebral cortex, where there are few DA reuptake pumps, via the inhibition of NE reuptake pumps (Cyberspace) which is believed to mediate reuptake of DA and $\mathrm{NE}^{14}$. However, duloxetine has no significant affinity for dopaminergic, cholinergic, histaminergic, opioid, glutamate, and gamma-Aminobutyric $\operatorname{acid(GABA)}$ reuptake transporters so it could be considered as a selective reuptake inhibitor at the 5-HT and NE transporters. Duloxetine undergoes extensive metabolism, but the major circulating metabolites do not significantly contributed to the pharmacologic activity ${ }^{15}$.

Major depressive disorder is believed to be due to an increase in pro-inflammatory cytokines within the central nervous system. Antidepressants that have a similar mechanism of action as duloxetine, i.e. 5-hydroxytryptamine metabolism inhibition, cause a decrease in proinflammatory cytokine activity and increase in anti-inflammatory cytokine; this mechanism may apply to duloxetine in its effect on depression but research on cytokine specific to duloxetine therapy is not avaliable ${ }^{16}$.

Duloxetine used as an analgesic for treating diabetic neuropathy and central pain syndrome such as fibromyalgia due to its sodium ion channel blockade ability $^{17}$. 


\section{MATERIAL AND METHODS}

Forty male albino rats weighing $180-200$ gr body weight were used in this investigation. The animals were divided into two groups as following:

Group I: were 20 animals served as control and received vechile daily

Group II: were 20 animals and received duloxetine in a dose of $10 \mathrm{mg} / \mathrm{kg}$ body weight that dissolved in distilled water and administrated through the metallic curved oropharngeal tube ${ }^{2}$.

The animals were caged, five animals per cage, they were maintained under optimum conditions of good ventilation and temperature in animal house of faculty of dentistry, Suez Canal University. Animals were supplied natural diet and drinking water adlibitum, through the whole experiment periods which lasts for 3 months.

At the end of the experiments, which lasts for 3 months, half of the animals of different groups (group I.1 and II.1) were sacrificed by cervical dislocation. The rest of animals (group I.2 and II.2) were allowed for a recovery period of 30 days.

At the end of recovery period, the rest of animals (groups I.2 and II.2) were sacrificed also by cervical dislocation.

Tongues of animals of different group were dissected out, cut longitudinally into two halves, fixed in $10 \%$ neutral buffered formalin, processed to be embedded in paraffin. Six microns thick sections were cut to be stained with:

1- Hematoxylin and eosin for histological examination.

2- Immunohistochemical localization of $\mathrm{E}$ cadherin

Small sized $\left(1 \mathrm{~mm}^{3}\right)$ specimens from the tongue of the animals of different groups was rapidly fixed in 3\% phosphate buffered glutraldehyde ( $\mathrm{PH} 7.2)$ for 1-2 hours (primary fixation) and prepared for electron microscope examination that was carried out using the T.E.M. (JEOL 1000) in the faculty of Agriculture, Cairo university.

\section{RESULTS}

\section{I-Light microscopic results:}

\section{A - Haematoxylin and Eosin results:}

Control group (Group I.1 \& I.2): The histological examination of the mucous membrane of the tongue of the rats showed the normal histological features of surface epithelium and underlying lamina propria. The dorsal surface of the tongue showed the different types of papillae including the filiform, fungiform papillae and circumvallate papillae, and lingual glands showed normal histological architecture Fig (1)

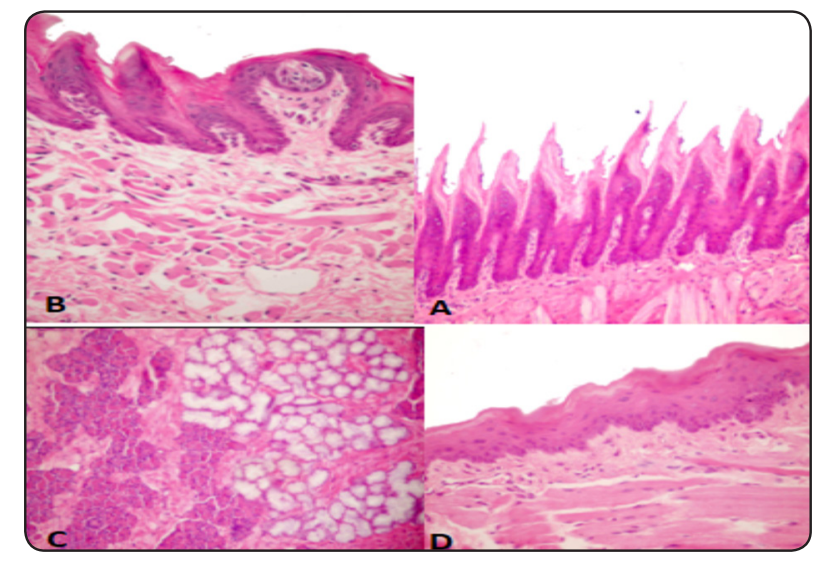

Fig. (1) A photomicrograph of tongue of a control rat showing (A) dorsal surface of the tongue of control with filliform papillae (B) fungiform papilla with taste bud at its superior position (C)lingual salivary gland serous acini and mucous acini (D) the mucous membrane of the ventral surfaces $\{\mathrm{H} \& \mathrm{E}$, orig. mag. 250, 400, 250, 250\}.

Group II.1: light microscopic examination of rats received daily dose duloxetine of $10 \mathrm{mg} / \mathrm{kg}$ of body weight dissolved in distilled water for 3 months showed degenerative changes in the dorsal, ventral and their lingual glands.

Filliform papillae showed deformation with decrease in the length and number, atrophic epithelial covering and loose normal shape of their epithelial ridge. Fungiform and circumvallate papillae showed atrophic epithelial surface, hyperkeratinization of epithelial covering was a marked result after Duloxetine treatment. lamina propria and showed 
shrunken and atrophied taste buds that may be completely lost. The underlying lamina propria showed dissociation of their collagen fiber. Lingual salivary glands showed degree of degeneration represented by cytoplasmic vacuolization of their serous acinar cells while their mucous acinar cells showed cystic transformation, the ductal cells showed flattening of their lining and stagnation of secretion in their lumen. Ventral surface of the tongue of group II.1 showed atrophic changes represented by thin epithelial with intercellular cytoplasmic vacuolization and their lamina propria showed fibers dissociation with blood vessels dilatation that engorged with RBCs. Fig (2)

Group II.2: examination of the tongue of rats treated with duloxetine for 3 months and then left for a recovery period for one month showed marked improvements in their histological structure

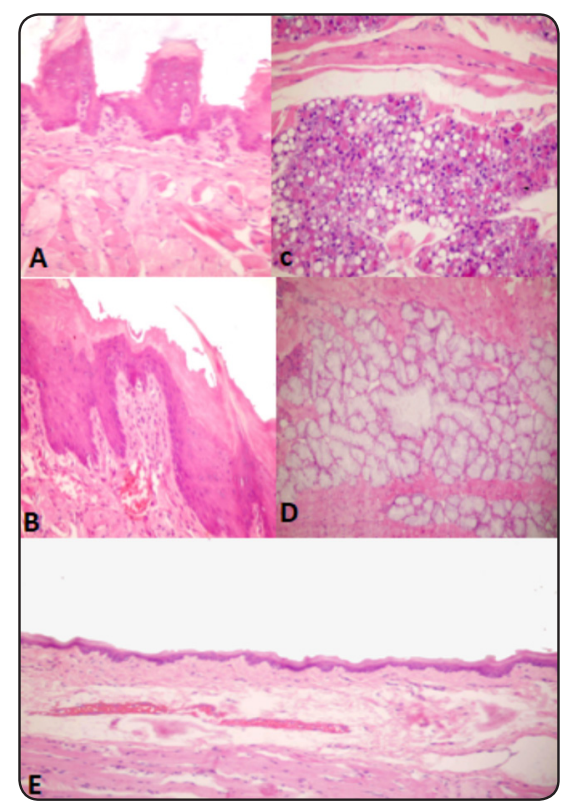

Fig. (2) A photomicrograph of group II.1 rats showing (A) atrophied and deformed filliform papillae with areas of hyperkeratosis (B) atrophied fungi papillae with degenerated taste bud and hemorrhage in underlying lamina propria(C) serous acinar cells with severe cytoplasmic vacuolization (D) mucous acinar cells with cystic transformation (E) ventral surfaces of tongue showing thin epithelial surface with disassociation and degeneration of under laying collagen fibers of lamina propria and dilated blood vessels $\{\mathrm{H} \& \mathrm{E}$, orig. mag. $250,250,400,250,250\}$ represented by normal thickness of surface epithelium, with some areas of hyperkeratinization, normal epithelial ridges and less cytoplasmic vacuolization.

Fungiform and circumvallate papillae showed normal architecture with almost normal taste buds. lingual salivary gland showed improvement represented by decrease in number and size of cytoplasmic vacuolization of their acinar cells some acinar cell showed complete absent of cytoplasmic vacuolization. Mucous cell showed normal appearance with little cystic transformation.

Ductal cells showed normal epithelial lining, some ducts showed stagnation of secretion in their lumen. Ventral surface showed normal epithelium thickness. Lamina propria showed normal collagen fibers with normal vascularity and cellularity. Fig (3)

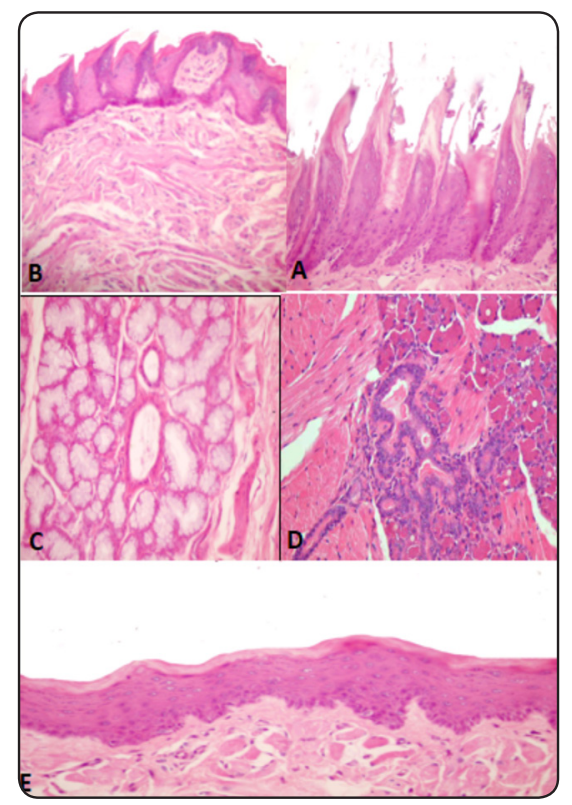

Fig (3): A photomicrograph of group II.2 rats showing (A) almost normal filiform papillae with normal epithelial thickness and normal underlying lamina propria. (B) fungiform papillae with normal taste bud but losing their normal mushroom shape and filiform papillae with some cytoplasmic vacuolization. (C)mucous acinar cells with a stagnant secretion in their ducts. (D) serous acinar cells with decreasing in cytoplasmic vacuolization and stagnant secretion of their ducts (E) normal surface epithelium and lamina propria of the ventral surfaces of the tongue $\{\mathrm{H} \& \mathrm{E}$, orig. mag. 400 , $250,400,250,250\}$. 


\section{Immunohistochemical localization of E-Cad- herin (Table 1)}

Examination of sections taken from the tongues of rats of control group (group (I.1, I.2) and incubated with mouse monoclonal antibody of E-cadherin clone 36B5 revealed a strongly positive reaction in the epithelial cells of the different strata of the surface epithelium of the dorsal and ventral surfaces of the tongue.

The lingual salivary glands revealed strongly positive staining reactivity both in the mucous and serous acinar and ductal cell (FIG 4)

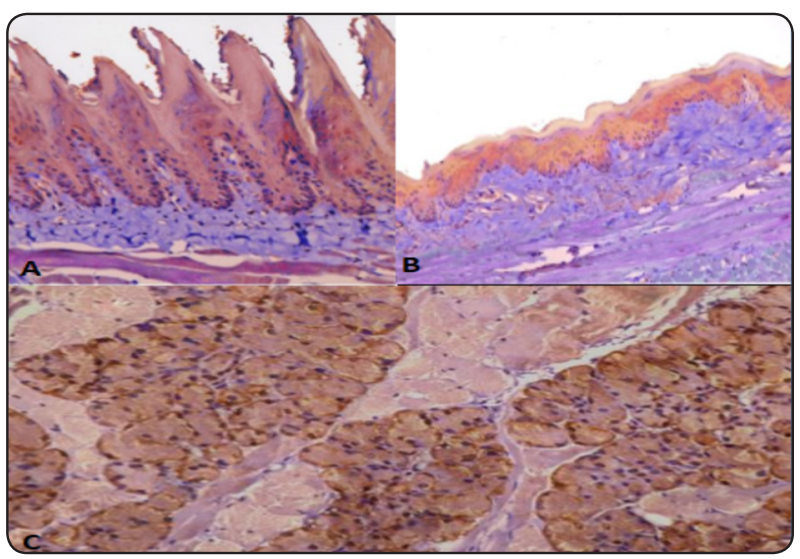

Fig (4) A photomicrograph of the tongue of control group incubated with mouse monoclonal antibody of E-cadherin clone 36B5 showing (A) strongly positive staining reactivity of the epithelial cells of dorsal surface of the tongue (B) strongly positive staining reactivity of the epithelial cells of ventral surface of the tongue $(\mathrm{C})$ strongly positive staining reactivity of the epithelial cells of ventral surface acinar cell of the tongue $\{\mathrm{H} \& \mathrm{E}$, orig. mag. 400, 250, 400\}.

The tongue obtained from group II.1 animals treated with a daily dose of duloxetine of $10 \mathrm{mg} /$ $\mathrm{kg}$ of body weight dissolved in distilled water for 3 months and incubated with mouse monoclonal antibody of E-cadherin clone 36B5 showed: Weakly positive reaction of the epithelial cells of the different strata of the surface epithelium of the dorsal and ventral surfaces of the tongue. Lingual salivary glands revealed weakly positive staining reactivity in the acinar and ductal cells of both the mucous and serous glands. Fig (5)

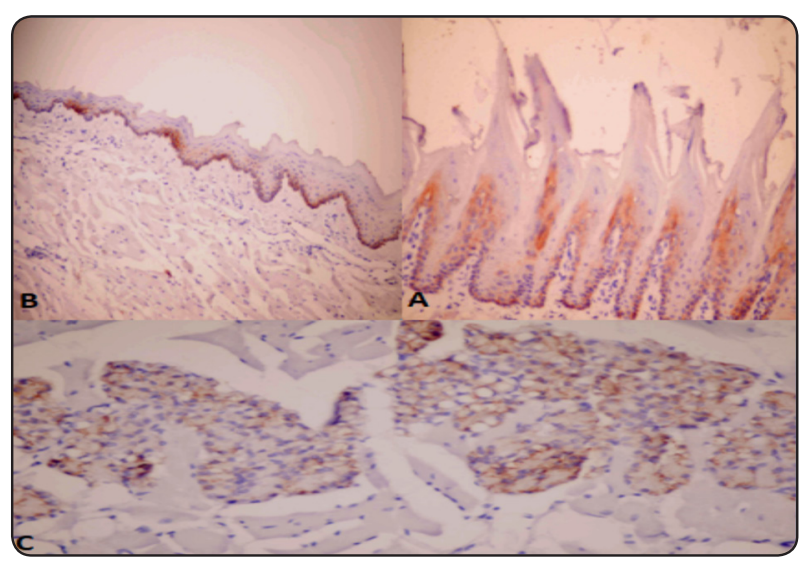

Fig (5) A photomicrograph of the tongue of group II.1 rats incubated with mouse monoclonal antibody of E-cadherin clone 36B5 showing (A) weakly positive staining reactivity of the epithelial cells of dorsal surface of the tongue (B) weakly positive staining reactivity of the epithelial cells of ventral surface of the tongue $(\mathrm{C})$ weakly positive staining reactivity of the epithelial cells of ventral surface acinar cell of the tongue $\{\mathrm{H} \& \mathrm{E}$, orig. mag. 250, 400, 250\}.

Group II.2 animals treated with duloxetine for 3 months and then left for a recovery period for one month showed moderately positive reaction to mouse monoclonal antibody of E-cadherin clone 36B5 in the epithelial cells of the different strata of the surface epithelium of the dorsal, ventral surfaces of the tongue and lingual salivary gland (ducts and acini). Fig 6.

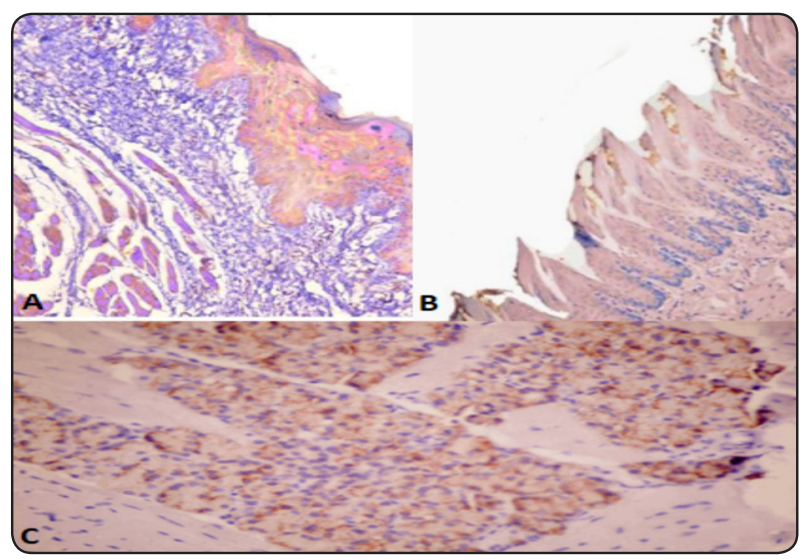

Fig (6) A photomicrograph of the tongue of recovery group incubated with mouse monoclonal antibody of E-cadherin clone 36 B5 showing (A) moderately positive staining reactivity of the epithelial cells of dorsal surface of the tongue (B) moderately positive staining reactivity of the epithelial cells of ventral surface of the tongue (C) moderately positive staining reactivity of the epithelial cells of ventral surface acinar cell of the tongue $\{\mathrm{H} \& \mathrm{E}$, orig. mag. $250,250,250\}$. 
TABLE (1) Illustrates the staining reactivity of the surface epithelium of the dorsal and ventral surfaces of the tongue as well as the acini and ducts of lingual glands of the different groups to E-cadherin

\begin{tabular}{|c|c|c|c|c|}
\hline \multirow{2}{*}{ Group } & \multirow{2}{*}{ Dorsal } & \multirow{2}{*}{ Ventral } & \multicolumn{2}{|c|}{$\begin{array}{c}\text { Lingual salivary } \\
\text { glands }\end{array}$} \\
\cline { 3 - 5 } & & & Acini & Ducts \\
\hline Control (I.1, I.2) & +++ & +++ & +++ & +++ \\
\hline Group II.1 & + & + & + & + \\
\hline Group II.2 & ++ & ++ & ++ & ++ \\
\hline
\end{tabular}

Scores of staining reaction:

- Negative

$+\quad$ Weakly positive

+ Moderately positive

++ Strongly positive

\section{Transmission Electron-microscopic results}

The ultrastructure of the dorsal surface of the tongue of the control group (I.1, II.2) showed different layers of keratinized stratified squamous epithelium that protrude over the surface to form the filliform and fungiform papillae.

The basal layer composed of one row of low columnar cells. The nuclei of basal cells were oval and possessed shallow invaginations of the nuclear envelope. At the lateral and superficial boundaries of the basal cells, the plasma membrane formed highly irregular convoluted interdigitaions with adjacent cells. Adjacent membranes were connected by desmosomes of variable lengths

The basal cells were separated from the underlying connective tissue by continuous and uninterrupted basal lamina. It was composed of electron lucent zone, lamina lucida, electron dense zone, lamina densa, and anchoring fibrils forming the reticular lamina. The basal cells were connected to basal lamina by spotted and numerous hemidesmosomes. All types of cell organelles including mitochondria, smooth and rough endoplasmic reticulum and Gologi bodies were found.
The spinous cell layer was composed of several rows of polyhedral cells with spherical nuclei and prominent nucleoli. The nuclear membrane had shallow indentations. The plasma membrane of spinous cells was connected together by desmosomes. Odland bodies were sometimes observed as intracytoplasmic small oval internally lamellated structures in the upper spinous layers and were usually located adjacent to the cell membrane

The granular cell layer was composed of 2-3 layers of flattened cells with elongated flattened nuclei. Some granular cells also contained (Odland bodies) with electron dense lamellations. The cytoplasm contained few mitochondria and small circular keratohyaline granules. Desmosomes of granular cells were small and regularly distributed and the surface was covered by keratinous layer

Nonkeratinocytes at different level of epithelial cells were seen, Melanocytes appeared between basal cells extended their cytoplasmic processes into intercellular spaces of epithelial cell. Langerhans cells were found in upper layers of epithelial cells with their characteristic Bir_beck granule. Merkel cells demonstrated in between basal cells and showed desmosomal attachments with epithelial cells

The lamina propria was composed of collagen fibers, fibroblasts, small sized endothelial lined blood vessels which were embedded in an amorphous ground substance. The fibroblasts were the most prominent connective tissue cells which appeared spindle shaped with elongated nuclei. Few polymorphnuclear leukocytes, macrophages and lymphocytes were sometimes encountered. Fig (7)

Electron microscopic examination of the tongue samples of group II.1 were the rats treated with a daily dose duloxetine of $10 \mathrm{mg} / \mathrm{kg}$ of body weight dissolved in distilled water for 3 months showed marked degenerative changes. Variable sized cytoplasmic vacuoles were commonly observed in most of epithelial cells. Marked destruction of different 


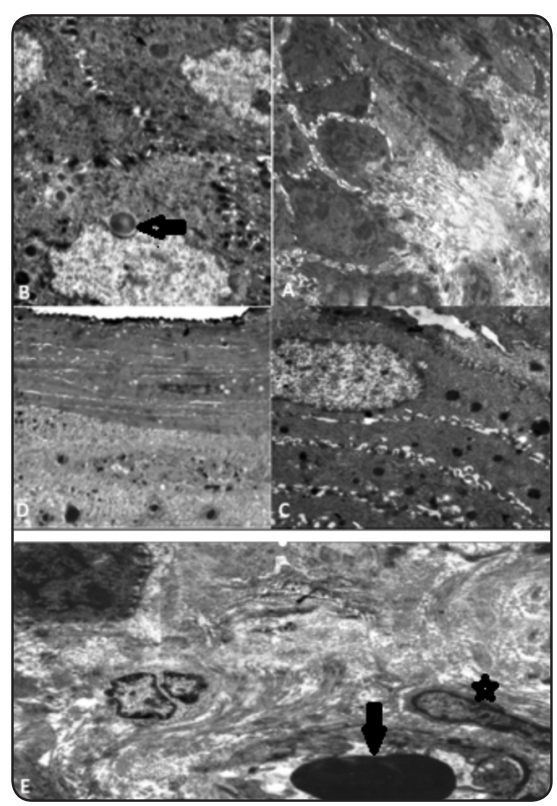

Fig. (7) An electron micrograph of the tongue of control animal showing (A) basal cell with small intercellular spaces and lateral interdigitation (desmosomes) between them, resting on an intact basal lamina and connected to it by hemidesmosomes. The nuclei are oval and have shallow invaginations (B) The spinous polyhedral cells with small interdigitation of its plasma membrane between them, and Odland bodies (arrow). (C) showing upper granular cell layer with keratohyalyine granules (D) lamina propria with spindle shaped fibroblast (star), blood vessels (arrow) containing RBCs, and surrounded by collagen fiber. Uranyl acetate\& Lead citrate (x1000, x2000, x2000, x2000, x2000)

cell organelles was a common finding. Decrease in the size and number of keratohyaline granules in granular cells were demonstrated. Inflammatory cells appeared in the extremely widened intercellular spaces between the epithelial cells. Keratin layer showed increase in its thickness. The lamina propria revealed fibroblasts presenting cytoplasmic vacuolization and dissociated collagen fibers. A number of inflammatory cells appeared within the lamina propria which presented markedly dilated blood vessels. Fig (8)

Electron microscopic examination of the tongue samples of group II.2 were the rats treated with a

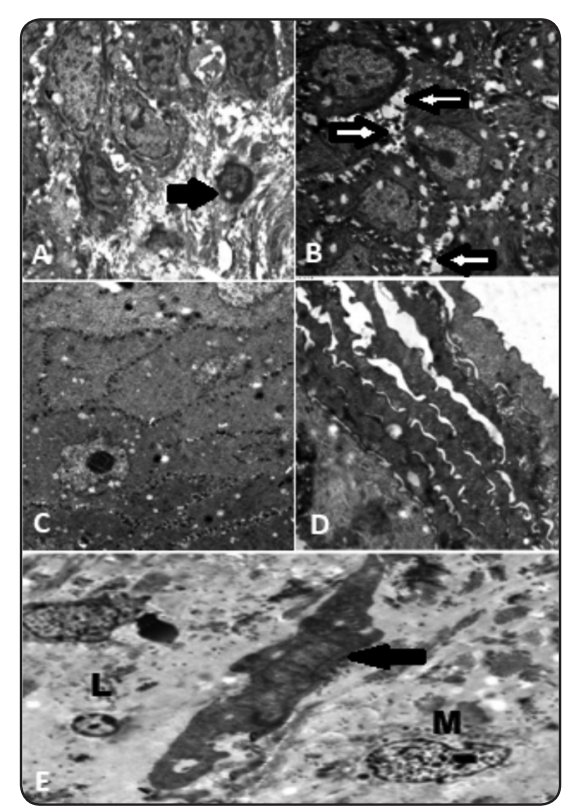

Fig (8) An electron micrograph of the tongue of group II.1 animals showing(A) basal cells with multiple cytoplasmic vacuoles and destruction of the underlying connective tissue with presence of lymphocyte (arrow) (B) spinous cell with multiple cytoplasmic vacuoles and widened intercellular spaces (arrows). (C) granular cell with multiple cytoplasmic vacuoles and decrease in the number of keratohyaline granules. (D) keratin layer with increase in its thickness and wide intercellular spaces between their layers. (E) lamina propria with fibroblast (arrow) showing cytoplasmic vacuolization, degenerated collagen fibers, lymphocyte (L), and macrophage (M). Uranyl acetate\& Lead citrate (x2000, x1000, x2000, x2000, x2000)

daily dose duloxetine of $10 \mathrm{mg} / \mathrm{kg}$ of body weight dissolved in distilled water for 3 months then left for 1 month for recovery showed improvement on ultrastructure level indicated by decrease in the cytoplasmic vacuolization in different epithelial cells layers. Normal desmosomal junction between epithelial cells. Almost normal size and no. of keratinohyaline granules in the granular layer, areas of normal thickness of keratin layer. Lamina propria showed normal ultrastructure view with almost normal fibroblasts that rested in amorphous ground substance and normal collagen fibers. Fig (9) 


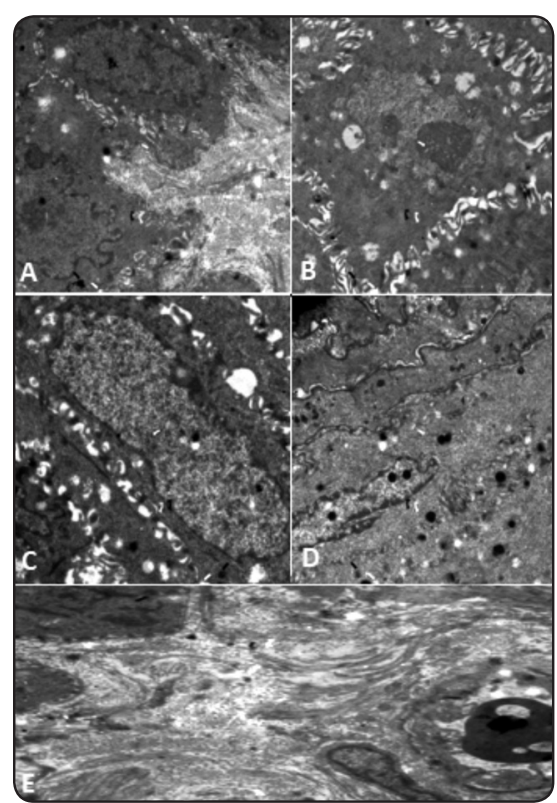

Fig (9) An electron micrograph of the tongue of group II. 2 animals showing (A) basal cells with normal ultrastructure picture (B) prickle cell with little cytoplasmic vacuolization (C) almost normal granular layer little cytoplasmic vacuolization (D) almost normal keratin layer and granular layer with keratohyaline granules and little cytoplasmic vacuolization (E) normal lamina propria and collagen fiber Uranyl acetate \& Lead citrate (x3000, x3000, x 3000, x2000, x2000)

\section{DISCUSSION}

Serotonin-norepinephrine reuptake inhibitors (SNRI) are used to treat depressive disorders and certain types of chronic pain ${ }^{18}$. Yoshida et al, 2017 reported that the common adverse effects of serotonin-norepinephrine reuptake inhibitors are nausea, dry mouth, dizziness and headache ${ }^{19}$. The histological examination of the lingual mucosa of rats that treated a daily dose of duloxetine of $10 \mathrm{mg} /$ $\mathrm{kg}$ of body weight dissolved in distilled water for 3 months revealed a degenerative change in the dorsal, ventral and their lingual glands. These degenerations manifested as atrophied epithelial surfaces, malformed papillae of dorsal surface of the tongue, taste buds of the fungiform and circumvallate were degenerated. Hyperkeratinization in some areas of surface epithelium was marked observation in our results.
Terminal differentiation is associated with increased keratohyaline granules and a reduction in nuclei in superficial layer cells. The amount of keratohyaline granules determines the extent of the keratinization process. The decrease or absence of keratohyaline granules is associated with abnormal terminal differentiation (parakeratinization) ${ }^{20}$.

According to the previous research hyperkeratosis of present investigation may be due to disturbance in of epithelial turnover rate due to the present degeneration that may affect the keratohyaline granules leading to abnormal desquamation and renewal of the surface epithelium causing the present hyperkeratosis.

Lamina propria showed dissociation of their fibers with marked dilatation of blood vessels. Lingual salivary glands showed severe cytoplasmic vacuolization of serous acinar cell and cystic transformation of mucous ones while their ducts showed thin epithelial lining with stagnation of secretion in their lumen.

All of these degenerative changes indicated that disturbance in cellular homeostasis and cellular failure and indicate that lingual mucosa suffered cellular failure as a result of administration of duloxetine.

The histological results are indicated by the transmission electron microscope results that revealed the degeneration upon the ultrastructural levels through destruction of the most cellular organelles degeneration, swollen mitochondria, increase in thickness of keratin and widening of intercellular spaces between epithelial cells lamina propria showed fibroblast with cytoplasmic vacuolization and Inflammatory cells observed at different levels of epithelial cells and lamina propria.

The appropriate regulation of cell-cell adhesion is an important event in the homoeostasis of different cell types. In epithelial cells, tight adhesion mediated by E-cadherin receptors is essential for the differentiation and functionality of epithelial sheets, upon assembly of cadherin-mediated cell- 
cell contacts ${ }^{21}$. Cadherins have been postulated to be responsible for the occurrence of contact inhibition of cell growth ${ }^{22}$.

In the present investigation, examination of lingual mucosae showed weakly positive reaction in the different strata of epithelium to monoclonal antibody of E-Cadherin. Also the serous and mucous lingual salivary glands of the tongue for both group II.1 animals compared to the controls.

These results indicate both microscopic and submicroscopic results of group II.1 animals. In group II.1 animals treated with duloxetine, the decrease in the expression of E-cadherin may be due to decrease in protein synthesis and in turn alteration in adhesion molecules development or due to cytotoxic effect of the drug supporting this hypothesis Stepan et al. (2009) ${ }^{23}$ supported this hypothesis and reported that Cadherin immunoexpression and normal function are not always in concordance. The function of E-cadherin may be altered because of the damage of other components of the intercellular adhesion systems, like cathenins. The author added that E-cadherin immunostaining intensity diminished with the degree of cell differentiation, or may be due to the cytotoxic effect of the drug.

McCorry, $2007{ }^{24}$ reported that antidepressants SNRI have an anticholinergic and antimuscarinic action, which blocks the action of parasympathetic system by inhibiting the effect of acetylcholine on the receptors of salivary glands. Thus dry mouth occurs, probably because the sympathetic portion independent nervous system prevails on the blocked parasympathetic system. Also khan et al., 200725 reported that duloxetine antagonizes the muscarinic receptor and causes anticholinergic adverse effects, such as dry mouth.

Olver $^{26}$ stated that the role of saliva in maintaining the oral health and even quality of life is obvious in people who are lacking sufficient saliva. Patient experiencing reduced salivary flow suffer considerable morbidity, including dental caries, mucosal infections, dysphagia and discomfort with problem with eating, speaking, swallowing and frequent disturbances in taste.

The mucous glycoprotein secreted by salivary gland such as MUC5B, MUC7 and proline rich glycoproteins, play a major role in lubricating oral tissues. This lubrication reduces trauma to soft tissue during mastication swallowing and speaking. The author added that bacterial and fungal infection may affect the oral mucosa as a result of decrease in salivary flow that lead to inflammation ${ }^{27}$.

According to previous researches, we suggested that the degenerative changes that occurred in the lingual mucosa after administration of duloxetine as a result of decreasing in the salivary flow of major salivary glands that leading to inflammation and infection of oral mucosa tissues, including lingual mucosa, due to decrease in lubrication effect of saliva.

The dilatation of blood vessels and their engorgement with blood was a constant feature in our results that may lead to stagnation and decrease of blood flow, resulting in hypoxia and ischemia which in our opinion may be an additional cause of structural deterioration of the tissue of the oral mucosa.

We also should also consider the adverse direct toxic effect of the duloxetine drug on the oral mucosal tissue that may lead to the present degeneration.

The examination of the lingual mucosa of the rats that were allowed a recovery period of 30 day revealed that a degree of improvement upon microscopic and submicrscopic levels. These improvements presented as decrease in number of cytoplasmic vacuolization in the surface epithelium, tongue papillae restore their normal shape, return of normal epithelial thickness of ventral and dorsal surfaces and the lamina propria return its normal texture. 
Lingual salivary glands showed almost normal appearance except for some areas of cytoplasmic vacuolization and some ducts showed dilatation of their lumen with stagnant secretions.

The e-cadherin results of group II.2 were moderately positive indicating the partial improvement of cell-cell adhesion between cells which reflect the general improvement occurred in the lingual mucosal tissue.

In our opinion failure of complete recovery of the lingual mucosa after 30 days may be due to insufficient recovery period, complete regeneration might have needed more time.

According to pervious investigations, using duloxetine for treatment depressive disorders should be under supervision after weighing the possible risks and benefits

\section{REFERENCES}

1. Starr LR, Hammen C, Connolly NP, Brennan PA. Does relational dysfunction mediate the association between anxiety disorders and later depression? Testing an interpersonal model of comorbidity. Depress Anxiety. 31:7786. 2014

2. Patel S Kale P Addepalli V, Sarkar A and Savai J: Effect of a combination of duloxetine with hydroxyzine on experimental models of anxiety in mice. Indian J Pharmacol. 47: 173-176. 2015

3. Baldwin DS, Pallanti S, Zwanzger P. Developing a European research network to address unmet needs in anxiety disorders. Neurosci Biobehav Rev. 37:2312-2317. 2013

4. Pate D.R. Feucht C., Brown K. Ramsay J. Pharmacological treatment of anxiety disorders in children and adolescents: a review for practitioners. Transl Pediatr.7: 23-35. 2018.

5. Weston C. Antidepressant drugs. In: Klykylo WM, Bowers R, Weston C. Green's child and adolescent clinical psychopharmacology. Philadelphia: Lippincott Williams and Wilkins, pp 186-257; 2014

6. Henry A, Kisicki MD, Varley C. Efficacy and safety of antidepressant drug treatment in children and adolescents. Mol Psychiatry. 17:1186-1193. 2012

7. Connolly SD, Bernstein GA; Work Group on Quality Issues. Practice parameter for the assessment and treatment of children and adolescents with anxiety disorders. J Am Acad Child Adolesc Psychiatry 46:267-283. 2007

8. Hershman DL, Lacchetti C, Dworkin RH. "Prevention and management of chemotherapy-induced peripheral neuropathy in survivors of adult cancers: American Society of Clinical Oncology clinical practice guideline". J. Clin. Oncol. 32 : 1941-1967. 2014

9. Sommer C, Häuser W, Alten R. Drug therapy of fibromyalgia syndrome. Systematic review, meta-analysis and guideline]". Schmerz (in German). 26: 297-310. 2012

10. Bril V, England J. Franklin G. Evidence-based guideline: treatment of painful diabetic neuropathy-report of the American Association of Neuromuscular and Electrodiagnostic Medicine, the American Academy of Neurology, and the American Academy of Physical Medicine \& Rehabilitation”. Muscle Nerve. 43: 910-917. 2011

11. Attal N, Cruccu G, Baron R, et al. (September 2010). "EFNS guidelines on the pharmacological treatment of neuropathic pain: revision”. Eur. J. Neurol. 17: 1113-1188. 2010

12. Hoffmann J. 11th European Headache Federation Congress jointly with 31 st Congress of the Italian Society for the Study of Headaches.The Journal of Headache and Pain 18:111-151, 2017

13. Chappell L., Kajdasz L, D’Souzan S, Moldofsky. “A 1-year safety and efficacy study of duloxetine in patients with fibromyalgia”. Clinical Journal of Pain. 25:365-375. 2009.

14. Stahl, S. (2013). Stahl's essential pharmacology, $4^{\text {th }}$ ed. Cambridge University Press, New York. p. 305, 308, 309.

15. Bymaster, FP; Lee, Knadler. "The dual transporter inhibitor duloxetine: a review of its preclinical pharmacology, pharmacokinetic profile, and clinical results in depression”. Curr Pharm Des. 11 : 1475-1493. 2005

16. De Berardis D, Conti CM, Serroni N, Moschetta FS, Olivieri L, Carano A, Salerno RM, Cavuto M, Farina B, Alessandrini M, Janiri L, Pozzi G, Di Giannantonio M; Conti; Serroni; Moschetta; Olivieri; Carano; Salerno; Cavuto; Farina; Alessandrini; Janiri; Pozzi; Di Giannantonio. "The effect of newer serotonin-noradrenalin antidepressants on cytokine production: a review of the current literature". Int J Immunopathol Pharmacol. 23: 417-422. 2010

17. Mousa SA. A Brief Review of the Pharmacology of Amitriptyline and Clinical Outcomes in Treating Fibromyalgia. Biomedicines. (2): 24-30. 2017

18. Khouzam H. psychopharmacology of chronic pain: a focus on antidepressants and atypical antipsychotics. Postgrad Med. 128:323-30. 2016 
19. Yoshida K. Fukuchi,T. and sugawara H. Dysosmia and dysgeusia associated with duloxetine. BMJ Case Rep. 6:1-32017

20. Li W, Marshall C, Mei L, Dzubow L, Schmults C, Dans M.): Srcasm modulates EGF and Src-kinasesignaling in keratinocytes. J. Biol Chem. 280: 6036-6046. 2005

21. Erasmus J., Aresta S., Nola S., Caron E. and Braga V. Newly formed E-cadherin contacts do not activate Cdc42 or induce filopodia protrusion in human keratinocytes. Biology of the Cell 102: 13-24. 2010

22. van Roy F and Berx G. The cell-cell adhesion molecule Ecadherin. Cell Mol Life Sci.; 65:3756-3788. 2008)

23. Stepan A., Margritescu CL. and Sim I. immunoexpression in dysplastic lesions and urothelial carcinomas of the blad- der, cadherin and p63. Romanian Journal of Morphology and Embryology, 50:461-465. 2009

24. McCorry L. Physiology of the Autonomic Nervous System. Am J Pharm Educ; 71: 78. 2007

25. Khan A., bose A., Alexopoulos G. Double blind comparison of escitalopram and duloxetine in the acute treatment of major depressive disorder. Clin Drug Investig.27:481-492. 2007

26. Olver i. xerostomia:acommon advers effect of drugs and radiation Aust. Prescr.:29:97-98. 2006

27. Dawes C. Salivary flow patterns and the health of hard and soft oral tissues. American Dental Association. JADA. 139: $185-245.2008$ 